\title{
Modélisation numérique du comportement hydraulique des caissons Jarlan
}

\author{
P. Gomi ${ }^{1}$, J.M. Rousset ${ }^{2}$, F. Ropert ${ }^{3}$ P. Sergent ${ }^{1}$ \\ ${ }^{1}$ Centre d'Etudes Techniques Maritimes et Fluviales, Compiègne, France, \\ tel: 03.44.92.60.76, email: patrick.gomi@equipement.gouv.fr \\ tel:03.44.92.60.30,email:philippe.sergent@equipement.gouv.fr \\ ${ }^{2}$ School of Mech. Eng., The University of Edinburgh, Ecosse, \\ tel: +44(0)131.650.5685,email:jrousset@eng.ed.ac.uk \\ ${ }^{3}$ Services Navigation de la Seine, Paris, France, \\ tel: 01.44.06.63.62,email: francois.ropert@equipement.gouv.fr
}

\section{Résumé}

Une modélisation numérique du comportement hydraulique des caissons Jarlan est réalisée par un modèle bidimensionnel vertical. Ce code 2DV est basé sur une généralisation des calculs de perte de charge en régime instationnaire. La validation de ce code est effectuée en comparant les coefficients de réflexion et de transmission calculés à ceux déterminés expérimentalement avec un écran perforé puis un caisson complet. Cette analyse démontre que ce modèle est d'ores et déjà utilisable à des fins d'ingénierie.

\begin{abstract}
The numerical simulation of the Jarlan caisson's hydraulic behaviour is carried out by a vertical two-dimensional code. This 2DV code is based on the generalised calculation of unsteady flow loss coefficients. The validation of this simulation is done comparing the calculated reflection and transmission coefficients with those from experiments, first using a single perforated screen, then a whole caisson. The study shows that this code may be already considered as a design tool.
\end{abstract}

\section{Introduction}

\subsection{Les ouvrages verticaux}

Comparées aux digues à talus, les digues verticales présentent notamment les avantages d'un encombrement réduit et d'une construction faiblement consommatrice de matériaux. En revanche ces ouvrages, dans leur configuration traditionnelle, souffrent de nombreux inconvénients, au premier rang desquels figure la réflexion quasi totale que leur présence génère.

Ce fort niveau de réflexion induit une augmentation de l'agitation au voisinage de l'ouvrage qui est susceptible de gêner la navigation et d'accroître les risques d'érosion des fonds en pied d'ouvrage. Il faut également souligner l'extrême intensité des efforts s'exerçant sur ces ouvrages en cas de déferlement au droit de la paroi verticale exposée aux houles extérieures (Oumeraci et coll., 2001). 
Les structures verticales dites semi-réfléchissantes réduisent la réflexion le plus souvent grâce aux jours pratiqués dans la face avant de l'ouvrage. Jarlan fut le premier à proposer une telle technique (Jarlan, 1965) et depuis le début des années 70, la majorité des grands ouvrages verticaux français ont été construits suivant son procédé.

\subsection{Les caissons Jarlan}

Le caisson Jarlan, construit en béton armé, comporte essentiellement une chambre, fermée à l'arrière par un mur plein et délimitée, à l'avant, par une paroi percée de trous circulaires d'un diamètre voisin du mètre, suivant une densité conférant à la paroi une porosité de l'ordre de $25 \%$. Si nous nous référons aux ouvrages français, la paroi perforée présente en général une épaisseur e également voisine du mètre, la largeur de la chambre n'excédant généralement pas la quinzaine de mètres (figure 1).

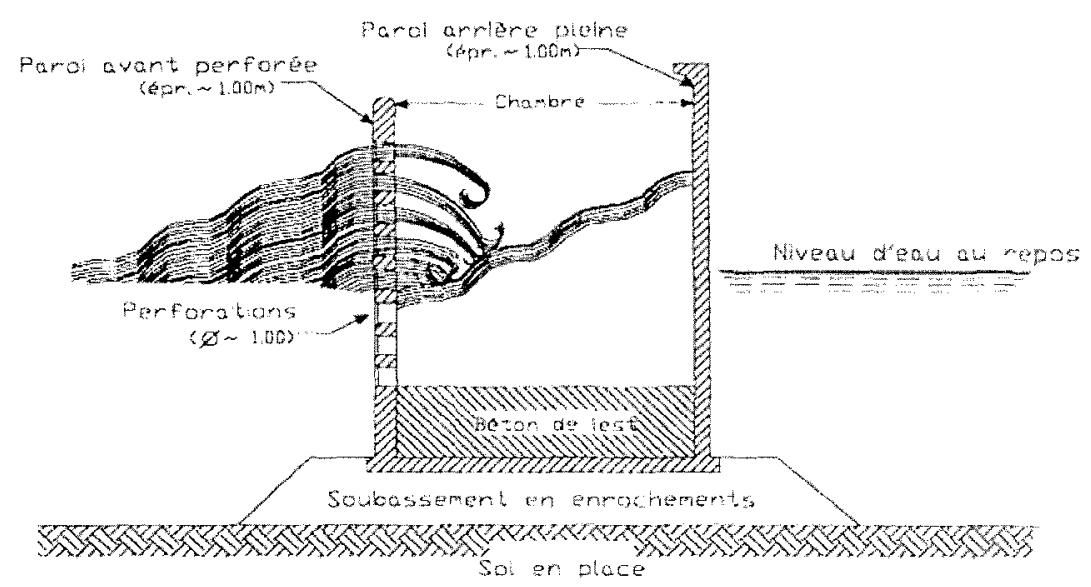

Fig. 1 : Perforated Jarlan caisson breakwater

La réduction des efforts globaux et l'abaissement de la réflexion sont les principaux avantages inhérents aux caissons Jarlan. Ils découlent de deux phénomènes interdépendants: la dissipation de l'énergie aux passages des vagues dans les perforations et le déphasage temporel des poussées horizontales sur les parois avant et arrière, abaissant ainsi l'effort global total sur la structure (Bélorgey et al., 1999).

Ces deux points concourent également à une limitation, d'une part des risques d'érosion et, d'autre part des franchissements au-dessus de la structure.

\subsection{Le dimensionnement}

Le processus de dimensionnement d'une digue est composé d'abord des calculs de stabilité générale des caissons sous l'action des houles, puis des calculs de stabilité interne (calculs de structure).

Généralement, l'ingénieur concevant ces ouvrages dispose de deux types d'outils d'études différents: les modèles réduits physiques hydrauliques particulièrement adaptés à la vérification de la stabilité générale de l'ouvrage et les modèles numériques de calculs de structure, le plus souvent aux éléments finis. Ces derniers sont capables 
de déterminer les contraintes dans le matériau afin de dimensionner les sections de béton armé, si l'on connaît les cas de charge pertinents.

Il s'est avéré, lors de la construction du dernier ouvrage français de type Jarlan construit à Dieppe au début des années 90 , que la définition de ces cas de charge constituait une difficulté majeure. Une solution à ce problème passait nécessairement par une schématisation du comportement hydraulique global des caissons Jarlan, en particulier afin de prendre en compte les houles obliques.

Ce projet a justifié la mise en place d'un programme national de recherche sur les caissons Jarlan associant le Ministère de la Recherche, l'université de Caen (faisant suite à celle du Havre à partir de 97), la SOGREAH et le CETMEF (ex-STCPMVN).

Des mesures expérimentales de la réflexion de la houle, de l'agitation à proximité des parois été conduites lors de modélisations physiques en cuve à houle (SOGREAH 1993) et en canal (Caminade 1992, Tabet-Aoul 1998, Rousset 2000). Lors de ces expériences, des mesures de vitesses des écoulements et de pression sur les parois ont également été réalisées. Parallèlement aux modélisations, des mesures sur le terrain ont été effectuées sur un caisson de la digue de Dieppe équipé en capteurs de pression (Rousset 1998). Enfin, le rôle du CETMEF a été, en association avec l'Université de Technologie de Compiègne, de proposer une modélisation numérique du comportement hydraulique des caissons Jarlan (Ropert 1999). C'est ce volet du programme que nous présentons dans cet article.

\section{L'approche générale}

\subsection{La modélisation recherchée}

L'objectif visé par la modélisation numérique recherchée est la capacité de simuler les variations du niveau de la surface libre du plan d'eau le long des parois du caisson, c'est à dire à l'intérieur et à l'extérieur de l'ouvrage, y compris sous l'influence des houles obliques, afin d'accéder, dans un second temps à la définition des cas de charge et des efforts en général.

Considérant un ouvrage placé dans son environnement réel, la modélisation globale des conditions auxquelles les structures sont soumises, devra prendre en considération plusieurs phénomènes se produisant simultanément. Il s'agit, en particulier des phénomènes de réfraction de la houle due aux variations des fonds marins, de diffraction ou d'atténuation derrière et en extrémité d'ouvrage, et de la réflexion. Concernant les caissons, il est nécessaire de modéliser la réflexion sur les diverses faces des parois concernées, la transmission des vagues à travers la paroi perforée et la dissipation au voisinage du mur ajouré. La prise en compte de l'influence de l'écoulement à l'intérieur du soubassement est également considérée.

\subsection{La stratégie adoptée}

Le CETMEF disposait déjà d'un modèle, REFONDE, capable de simuler les modifications de la houle dans sa propagation, c'est à dire les phénomènes généraux dans la zone extérieure à l'ouvrage. Dans le processus complet des calculs de dimensionnement interviendrait forcément le couplage avec 
REFONDE. C'est donc tout naturellement que nous avons eu tendance à privilégier une approche physique s'apparentant nettement à celle adoptée pour l'élaboration de ce dernier, en particulier en nous rapprochant autant que possible des modèles à potentiel.

Notre souci premier étant d'aboutir à un modèle facilement exploitable à des fins d'ingénierie, nous avons opté d'emblée pour un schéma simplifié où les phénomènes réels seraient non pas décrits finement, mais pris en compte à travers leur influence globale.

Ainsi les dissipations d'énergie induites par la turbulence générée dans les tourbillons d'expansion des jets sont elles représentées, d'une part, comme si elles se produisaient au voisinage immédiat de la paroi et, d'autre part, en quantité moyennée sur une période. Par ailleurs ces dispositions sont de nature à faciliter grandement le couplage avec le modèle REFONDE.

Nous avons donc développé des modèles stationnaires traitant une houle monochromatique. Le travail a porté sur la construction de trois modèles différents :

- Le premier modèle, bidimensionnel vertical (2DV), ne considère que les houles frontales, mais il renseigne sur la distribution de l'hydraulique dans la verticale. Il permet notamment d'étudier l'influence de l'épaisseur du lest ou encore celle de la géométrie d'un soubassement imperméable. C'est ce modèle que nous développons dans la partie suivante.

- Le second modèle (SOL), également bidimensionnel vertical, a été développé pour tenir compte des soubassements perméables en se basant sur l'équation de Forschheimer (1901). Le code est capable de simuler les écoulements dans les matériaux pulvérulents induits par une houle monochromatique en considérant, comme précédemment, la dissipation moyennée sur une période. Les deux modèles verticaux autorisent un couplage aisé.

- L'exploitation de ces deux codes a permis de guider l'élaboration du troisième modèle, bidimensionnel horizontal $(2 \mathrm{DH})$, basé sur l'équation de Berkhoff (ou «Mid Slope Equation ») (1976). Ce modèle représente l'outil recherché capable de traiter les houles obliques agissant sur une digue Jarlan située dans son environnement complet.

Concernant les deux derniers modèles (SOL et $2 \mathrm{DH}$ ), les lecteurs peuvent se reporter à la thèse de F. Ropert (1999) dans laquelle leurs bases théoriques, leur développements et leurs validation sont détaillés.

\section{LE MODELE 2DV}

\subsection{Le fluide général}

A l'écart des perforations l'écoulement dans le fluide est simulé par un modèle classique à potentiel pour lequel nous rappelons brièvement les hypothèses.

Le fluide parfait, incompressible est animé d'un mouvement irrotationnel. La vitesse dérive d'un potentiel $\phi$ qui vérifie l'équation de Laplace : $\Delta \phi=0$.

Tous les paramètres physiques nous intéressant se déduisent de ce paramètre, en particulier la charge : 


$$
H_{c}=-\rho \cdot \frac{\partial \varphi}{\partial t} \text { (Intégrale d'Euler) }
$$

où $\rho$ est la masse volumique de l'eau.

Nous travaillons sur les modèles stationnaires, ce qui signifie que nous recherchons des solutions harmoniques caractérisées par une seule période $\mathrm{T}$. Le potentiel peut donc s'écrire sous la forme suivante, $\omega$ étant la pulsation $2 \pi / T$ :

$$
\phi=\phi_{1} \cos \omega t+\phi_{2} \sin \omega t
$$

ce qui permet de définir le potentiel en nombres complexes :

$$
\varphi=\phi_{1}+i \phi_{2} \text { et } \phi=\operatorname{Re}\left(\varphi e^{-i \omega t}\right)
$$

D'un point de vue pratique, l'élévation de la surface libre par exemple, se déduit de notre paramètre de calcul, moyennant l'hypothèse d'une vitesse modérée :

$$
\eta=\operatorname{Re}\left(i \frac{\omega}{g} \varphi e^{-i \omega t}\right)_{z=0}
$$

où g est l'accélération de la pesanteur.

\subsection{La paroi perforée}

La modélisation de l'influence de la paroi perforée sur la propagation de vagues s'inspire des estimations classiques de pertes de charge dues à des rétrécissements et à des élargissements brusques dans des conduites (Ropert 1999).

La figure ci-dessous illustre en perspective et en coupe, l'état de l'écoulement au voisinage d'une perforation, à un instant où il s'opère par exemple de la gauche vers la droite. L'espace est découpé en petits volumes centrés autour des perforations.
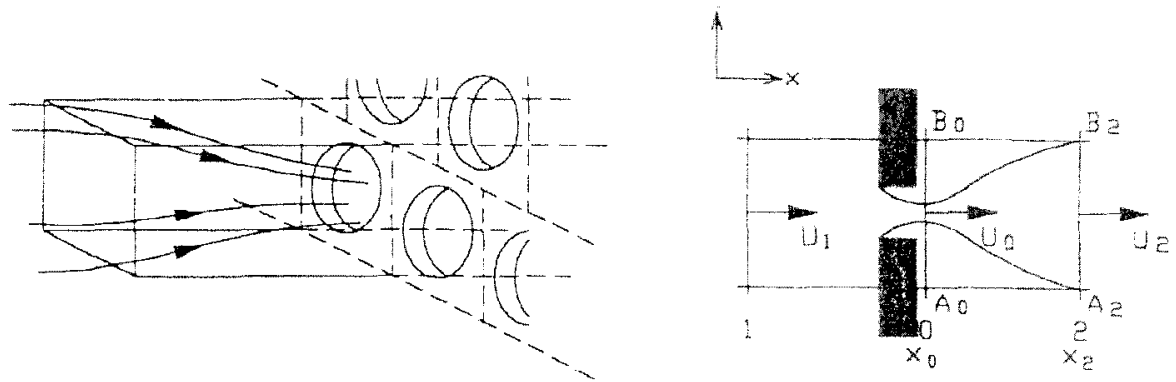

Fig. 2 : Flow streaming through perforations

L'écoulement amont, entre les sections 1 et 0 concerne un fluide considéré parfait et irrotationnel pour lequel l'intégrale d'Euler est vérifiée. Ce n'est évidemment pas le cas pour l'écoulement aval, entre les sections 0 et 2 où est appliqué le théorème de la quantité de mouvement, la difficulté résultant de la nature non stationnaire de l'écoulement et des débits non nuls à travers les frontières haute $\mathrm{B}_{0} \mathrm{~B}_{2}$ et basse $\mathrm{A}_{0} \mathrm{~A}_{2}$ du domaine $\mathrm{A}_{0} \mathrm{~B}_{0} \mathrm{~B}_{2} \mathrm{~A}_{2}$ concerné.

L'évaluation des termes s'opère en référence à un mouvement équivalent en débit et qui concernerait un fluide parfait irrotationnel. Les développements font apparaître une expression de déficit de charge en fonction de la vitesse dans les perforations, 
de sa dérivée temporelle et d'une longueur additionnelle représentative d'un éventuel retard de propagation des ondes (Ropert, 1999). En localisant ce déficit au plus près de la paroi perforée, il est possible de parvenir à l'expression suivante :

où :

$$
\frac{\partial \phi_{D}}{\partial t}-\frac{\partial \phi_{G}}{\partial t}=\frac{u_{0}^{2}}{2}\left(1-C_{C} m\right)^{2}+\frac{\partial u_{0}}{\partial t} \Delta \ell
$$

$\phi_{\mathrm{D}}$ et $\phi_{\mathrm{G}}$ représentent respectivement les potentiels réels à droite et à gauche de la paroi ;

$\mathrm{u}_{0}$ est la vitesse à l'intérieur de la perforation ;

$\mathrm{C}_{\mathrm{C}}$ est le coefficient de contraction de la veine liquide ;

$\mathrm{m}$ est la porosité de la paroi ;

$\Delta$ l la longueur additionnelle mentionnée plus haut.

S'agissant d'un écoulement alterné, il importe de rétablir la symétrie. Il suffit pour cela de remplacer $u_{0}^{2}$ par $u_{0}\left|u_{0}\right|$, terme qui est ensuite linéarisé en faisant des équivalences en termes de dissipation de flux d'énergie. Ces considérations conduisent finalement à l'expression suivante reliant les potentiels complexes de part et d'autre de la paroi et la vitesse complexe dans la perforation :

$$
\frac{\partial \varphi}{\partial x}=\frac{\varphi_{D}-\varphi_{G}}{\ell+i d}
$$

où $\mathrm{d}$ est le terme représentant la dissipation moyennée sur une période :

$$
d=\frac{1}{2 \omega} \cdot \frac{8}{3 \pi}\left(\frac{1}{C_{C}}-m\right)^{2}\left|\frac{\partial \varphi}{\partial x}\right|
$$

$\operatorname{avec} \ell=\mathrm{e}+\Delta \ell$, soit une longueur du même ordre que l'épaisseur de la paroi.

\subsection{Modélisation complète}

La modélisation complète du problème bidimensionnel vertical est réalisée à partir des relations précédentes. Le domaine de calcul, représentant une coupe verticale est schématisé par la figure ci-dessous : 


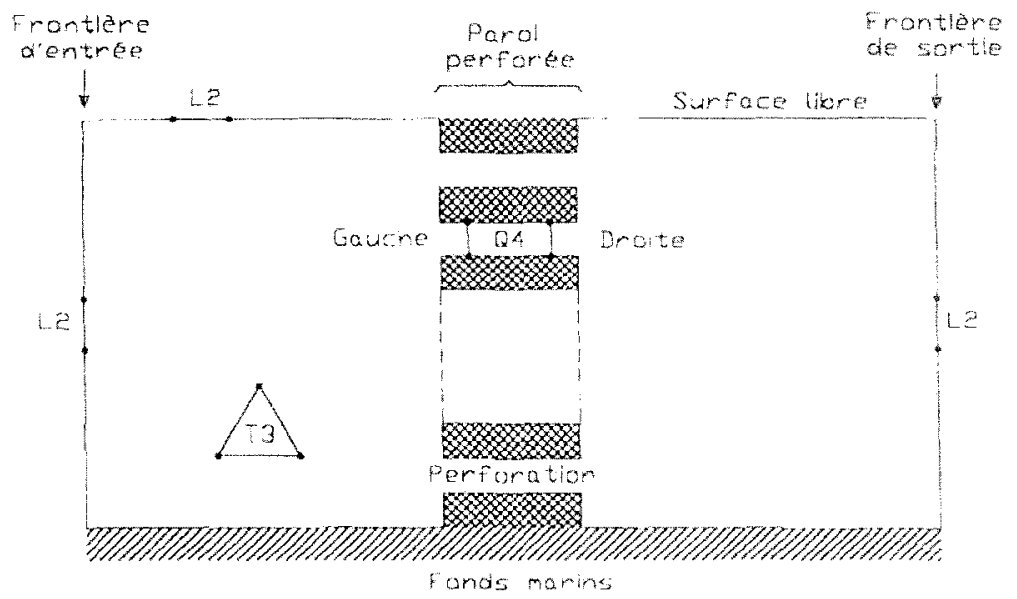

Fig. 3 : 2DV vertical modelling

La définition du problème complet requiert de préciser les conditions aux limites sur l'ensemble des frontières, à savoir :

fonds et paroi de béton $\frac{\partial \varphi}{\partial n}=0$

surface libre $\frac{\partial \varphi}{\partial n}=\frac{\omega^{2}}{g} \varphi$

frontière d'entrée $\frac{\partial \varphi}{\partial n}=i k \varphi-2 i k \varphi_{i n c}$

frontière de sortie ouverte $\frac{\partial \varphi}{\partial n}=i k \varphi$

frontière de sortie fermée $\frac{\partial \varphi}{\partial n}=0$

où $\mathrm{k}$ est le nombre d'onde et $\varphi_{\text {inc }}$ le potentiel incident précisé en entrée du modèle.

La frontière de sortie peut notamment être la matérialisation du mur arrière d'un caisson Jarlan, elle est fermée et imperméable. Le problème est résolu aux éléments finis, c'est à dire que le calcul va s'effectuer sur un maillage composé de petits éléments à l'intérieur desquels la solution est approximée à l'aide des valeurs aux nœuds. Dans notre cas, ce sont des éléments standards linéaires qui ont été retenus, avec des T3 pour l'intérieur et des L2 sur les contours extérieurs (Fig. 3). Il a toutefois fallu mettre au point un élément Q4 spécifique de prise en compte de la condition de dissipation dans les perforations. La nature implicite de cette condition, le paramètre $d$ étant fonction de la norme de la vitesse, a rendu nécessaire une résolution par itérations successives. Précisons enfin que, s'agissant d'une modélisation bidimensionnelle verticale, ce ne sont pas exactement des trous qui sont représentés mais plutôt des fentes horizontales dont la hauteur est choisie en raisonnant à porosité équivalente.

\subsection{Validation du modèle}

Pour tester le comportement de ce modèle, la première étape a consisté en la comparaison des résultats du code $2 \mathrm{DV}$ avec des mesures expérimentales de 
coefficients de réflexion et de transmission.

La structure test mise en œuvre est une paroi perforée épaisse de un mètre et de porosité $\mathrm{m}=0,35$, placée dans une profondeur d'eau $\mathrm{h}=10 \mathrm{~m}$. La première série de mesures provient d'essais en canal à houle réalisés avec un modèle physique au $40^{\text {ème }}$ (Caminade, 1992). La deuxième série correspond aux formules empiriques données par Bergmann et Oumeraci (1999) à partir d'essais réalisés à l'échelle 1 à GWK (Hanovre).

La modélisation numérique s'est opérée sur un domaine s'étendant sur 5 mètres de part et d'autre de la paroi. Le maillage comportait environ 2000 nœuds pour un nombre d'éléments voisin de 4000 unités. Les calculs ont été effectués avec un coefficient de contraction de 0,65 . Les comparaisons entre modèles physiques et numérique ont été menées pour les périodes de 5, 7,9 et 11 secondes.

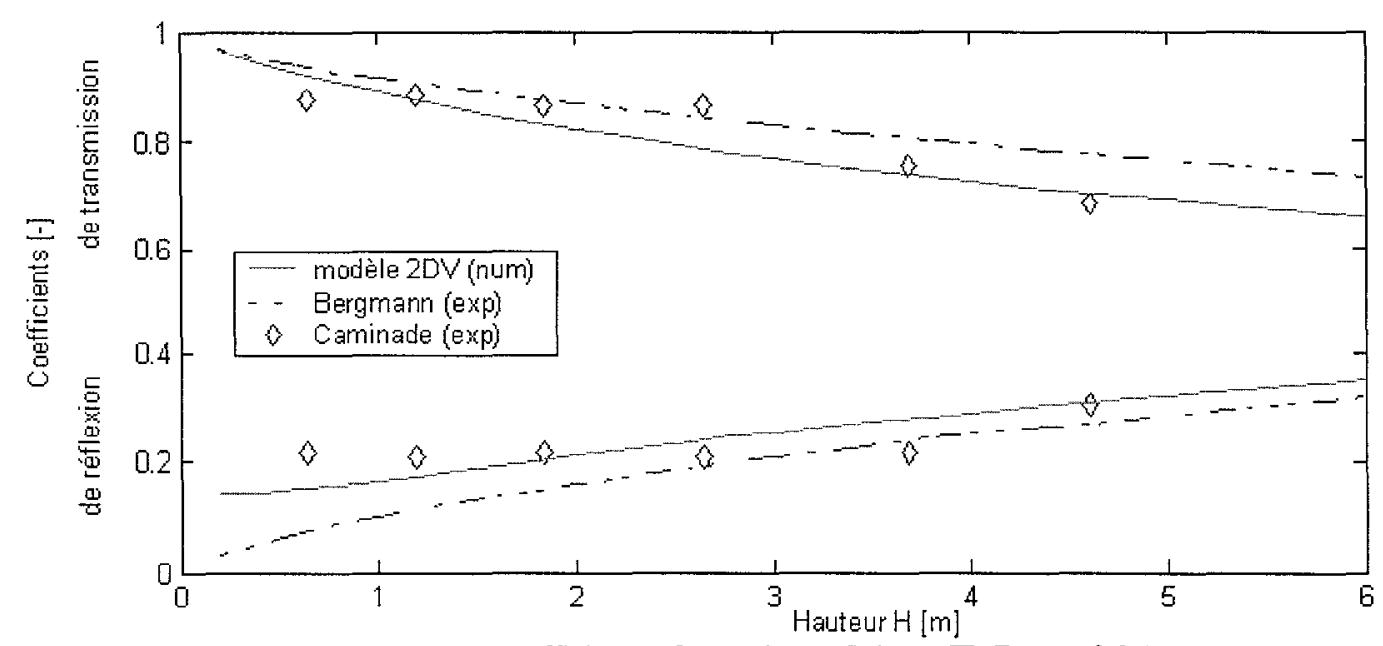

Fig. 4 : Hydraulic coefficients of a perforated sheet $(T=7 \mathrm{~s}, \mathrm{~m}=0,35)$.

La figure 4 représente la synthèse des résultats obtenus pour 7 secondes. Les lignes continues concernent les résultats numériques du modèle 2DV. Les points isolés et les lignes discontinues correspondent aux mesures expérimentales. La partie supérieure du graphique représente le coefficient de transmission et la partie inférieure le coefficient de réflexion. Les valeurs sont données en fonction de celle de la houle régulière incidente portée en abscisse en mètres.

Les écarts entre le modèle 2DV et les données expérimentales sont assez faibles. Elles sont en fait de l'ordre de l'incertitude des mesures. La concordance entre les résultats physiques et numériques apparait donc très satisfaisante sur cette configuration et il en va de même pour les autres périodes.

La validation se poursuit en mettant en cuvre une configuration proche de celle des caissons de Dieppe La largeur de chambre B est de 13,5 m, la profondeur devant la berme est de $10 \mathrm{~m}$ alors que la profondeur dans la chambre n'est que de $6,5 \mathrm{~m}$ (présence du lest). La porosité de la paroi est de $28 \%$.

Le maillage du domaine de calcul qui couvre toute la chambre et s'étend sur une dizaine de mètres devant l'ouvrage, se compose d'un assemblage de quelques 7600 éléments reliant environ 3900 nouds. 
Dans les figures suivantes, le coefficient de réflexion globale de la structure calculé par le modèle 2DV est comparé aux données expérimentales de Rousset et al (2000). Ces mesures ont été réalisées en canal à houle, en houle régulière, la structure étant reproduite à l'échelle du $25^{\mathrm{ème}}$. Les cambrures $\gamma=\mathrm{H} / \mathrm{L}$ des houles générées varient entre 0,5 et $5 \%$.

Sur la figure 5 , les valeurs de la réflexion sont données en fonction de la largeur relative de la chambre $\mathrm{B} / \mathrm{L}$, $\mathrm{L}$ étant la longueur d'onde incidente. Les deux courbes continue et discontinue montrent l'évolution des résultats du modèle $2 \mathrm{DV}$ pour deux cambrures de 2 et $4 \%$. Nous avons caler la formule de dissipation en fonction des observations sur la longueur des jets faites par Rousset (2000) en considérant une longueur additionnelle équivalente à l'épaisseur de l'écran (1 m).

Les optima de rendement du caisson, i.e. les minima du coefficient de réflexion, sont bien obtenus pour des largeurs relatives voisines, entre 0,15 et 0,20. De façon générale nous pouvons noter une bonne concordance des résultats du modèle 2DV avec les mesures expérimentales.

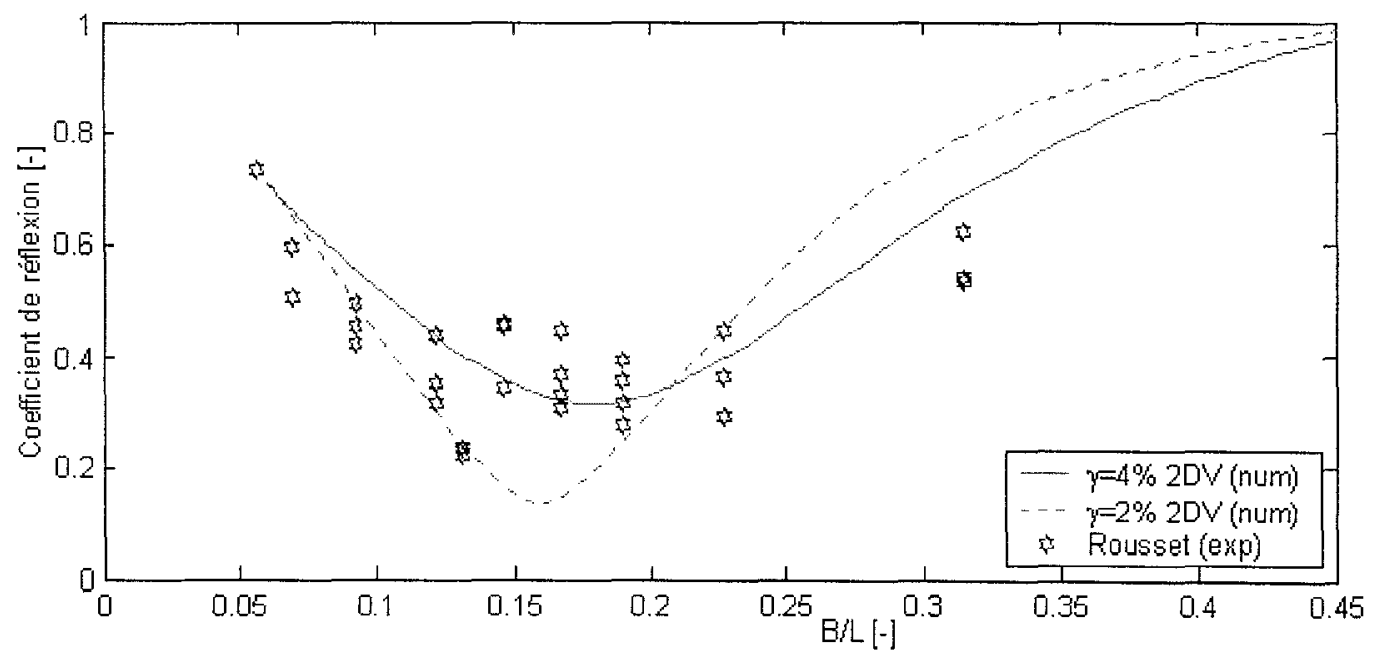

Fig. 5 : Comparison of global reflexion coefficient for a perforated Jarlan caisson breakwater

La concordance est encore améliorée si nous comparons les résultats du modèle 2DV avec les coefficients de réflexion expérimentaux calculés pour la fréquence fondamentale $1 / \mathrm{T}$ de la houle. En ne prenant pas en compte les harmoniques de rangs supérieurs générés par le caisson (au contraire des données expérimentales précédentes), nous sommes précisément dans les hypothèses du modèle numérique. La figure 6 présente les résultats des deux modélisations pour des cambrures identiques. 


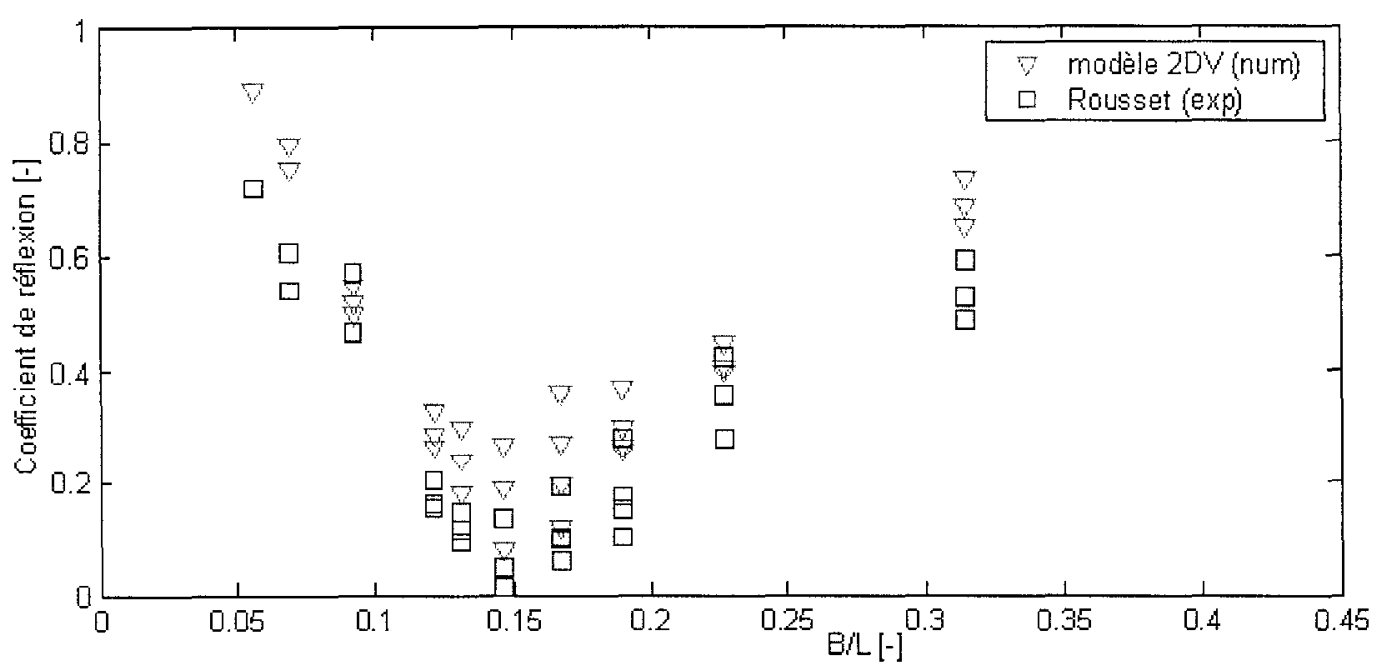

Fig, 6 : Comparison of reflexion coefficient for the fundamental period $T$

Le modèle 2DV décrit correctement la variation de la réflexion en fonction de la largeur relative $\mathrm{B} / \mathrm{L}$, bien qu'il tende à légèrement la surestimer. On notera en particulier que la plage du minimum de réflexion est identique.

Nous considérons donc que la modélisation numérique bidimensionnelle et verticale est validée. Les développements de ce code ont été repris dans la modélisation bidimensionnelle horizontale (2DH) et les comparaisons effectuées avec les mesures expérimentales montrent que ces deux modèles sont fiables (Ropert, 1999).

\section{Conclusion}

Le dimensionnement des caissons Jarlan passe nécessairement par une modélisation de leur comportement hydraulique. Une possibilité consiste à adopter un schéma physique simplifié où toute la dissipation s'opère dans le voisinage immédiat de la paroi. Les équations quantifiant ces phénomènes peuvent être obtenues par une généralisation des calculs de perte de charge en régime instationnaire appliqués au cas de la houle monochromatique.

Cette démarche s'est traduite par le développement d'un modèle vertical aux éléments finis dont l'exploitation a mis en évidence l'aptitude à décrire des phénomènes globaux comme la réflexion et la transmission d'une paroi perforée isolée ou encore la réflexion d'un caisson ainsi que l'agitation au droit de la paroi opaque.

Des améliorations de performance peuvent être obtenus à travers une meilleure connaissance de l'écoulement à travers la paroi perforée notamment. Mais c'est surtout la transformation du modèle en code instationnaire qui devrait apporter les bénéfices les plus significatifs, notamment dans la description des phénomènes locaux. Il restera, par la suite à exploiter les résultats en terme d'efforts et de pression, afin de proposer des formules encore plus adaptées que celles aujourd'hui couramment employées. Dans l'immédiat, nous considérons que les modèles 2DV, SOL et 2DH constituent en l'état des outils d'ores et déjà exploitables à des fins d'ingénierie. 


\section{Bibliographie}

Bélorgey M., Rousset JM., Tabet-Aoul EH, Bergmann H., de Gerloni M., Colombo D., Franco L. et Passoni G., 1999. "Perforated caisson breakwaters: wave loads and hydraulic performance", Proc. of Int. Conf. on Coastal Structures, Santander (Spain), Ed. Balkema, pp 603-612.

Bergmann H. et Oumeraci O. (1999). "Hydraulic performance of perforated structures", Proc. of the $5^{\text {th }}$ COPEDEC Conf., Cap Town (South Africa), $10 \mathrm{p}$.

Berkhoff J.C.W. (1976). "Mathematical models for simple harmonic linear water waves, wave refraction and refraction", Delft Publication $n^{\circ} 163,103 \mathrm{p}$.

Caminade D. (1992). "Interaction houle-structure. Etude de la transmission de la houle à travers une paroi perforée (type Jarlan)", thèse de Doctorat, Université du Havre.

Forchheimer P. (1901). "Wasserbewegung durch Bodem", Z. Ver. Deutsch. Ing., vol. 45, pp 1782-1788.

Jarlan G.E. (1965). "The application of accoustic theory to the reflective properties of coastal engineering structures", DME/NAE Quaterly Bulletin No 1965(1), National Research Council of Canada, Ottawa, pp 23-63.

Ropert F. (1999). "Modélisation numérique du comportement hydraulique des caissons Jarlan", thèse de Doctorat, Université de Technologie de Compiègne.

Rousset, J.-M. et Bélorgey, M. (1998). "Analyse des variations de pressions générées par la houle sur la digue Jarlan de Dieppe", Vèmes Journées Nationales Génie civil - Génie côtier, Ed. Paralia, pp 223-230.

Rousset, J.-M., Kohl T et Bélorgey, M. (2000). "Phénomènes dissipatifs dans une digue à paroi perforée", VIèmes Journées Nationales Génie civil - Génie côtier, Ed. Paralia, pp 307-314.

Rousset J.-M. (2000). "Sollicitations d'une digue Jarlan sous l'action de houles aléatoires - Cas du port de Dieppe", Thèse de Doctorat, Université de Caen.

SOGREAH (1993). "Etude du comportement des caissons à façade verticale perforée. Essais en bassin à houle", rapport n 51344, STCPMVN, Compiègne.

Tabet Aoul, E.H. (1998). "Etude du fonctionnement hydraulique des digues à paroi perforée", thèse de Doctorat, Université du Havre. 